\title{
Value of hepatic elastography and Doppler indexes for predictions of esophageal varices in liver cirrhosis
}

\author{
Adriana Bințințan ${ }^{1 *}$, Romeo Ioan Chira ${ }^{1 *}$, Vasile Virgil Bințințan², Georgiana Anca Nagy ${ }^{1}$, \\ Maria Roberta Mânzat-Săplăcan', Monica Lupșor-Platon ${ }^{3}$, Horia Ștefănescu ${ }^{4}$, \\ Maria Magdalena Duma ${ }^{5}$, Simona Doina Vălean'1, Petru Adrian Mircea1
}

\begin{abstract}
${ }^{1} 1{ }^{\text {st }}$ Medical Clinic, Gastroenterology Department, Emergency Clinical County Hospital, ${ }^{2} 1{ }^{\text {st }}$ Surgical Department, Emergency Clinical County Hospital, ${ }^{3}$ Medical Imaging Department, Regional Institute of Gastroenterology and Hepatology "Prof. Dr. Octavian Fodor" ${ }^{4}$ Hepatology Department, Regional Institute of Gastroenterology and Hepatology "Prof. Dr. Octavian Fodor", ${ }^{5}$ Radiology Department, Emergency Clinical County Hospital "Iuliu Hațieganu" University of Medicine and Pharmacy, Cluj-Napoca, România
\end{abstract}

*the authors shared the first authorship

\begin{abstract}
Aims: Non-invasive methods are required to diagnose presence and grading of esophageal varices in patients with hepatic cirrhosis and in this respect we have evaluated the role of transient elastography and abdominal ultrasound parameters. Material and methods: Cirrhotic patients were prospectively evaluated by transient elastography and Doppler ultrasound for diagnosis of presence and grading of esophageal varices, the results being compared with the findings of the esophagogastroduodenoscopy. Results: Sixty patients with hepatic cirrhosis were analysed. The parameters that reached statistical significance for diagnosis of esophageal varices were: liver stiffness $(\mathrm{LSM})>15 \mathrm{kPa}$, hemodynamic liver index $(\mathrm{PVr} 1) \geq 0.66$, portal vascular resistance $(P V R)>17.66$ and splenoportal index $(\mathrm{SPI})>4.77$. The only parameter that reached statistical power for the diagnosis of large esophageal varices was LSM at a cut-off value of $28.8 \mathrm{kPa}$. Conclusions: Assessment of LSM in patients with liver cirrhosis can predict both the presence of esophageal varices and of large esophageal varices. The PVr1, PVR and SPI Doppler indexes can be used to diagnose the presence of esophageal varices but have no role in the prediction of large esophageal varices. Further studies are required to confirm these results and offer a stronger clinical significance.

Keywords: esophageal varices, esophagogastroduodenoscopy, Doppler ultrasound, non-invasive diagnosis, transient elastography
\end{abstract}

\section{Introduction}

Esophageal varices (EV) are present in $40 \%$ of patients with compensated liver cirrhosis and in $60 \%$ of those with decompensated disease, having a constantly progressive evolution; once discovered they need to be under constant surveilance [1]. The annual rate of in-

Received 11.12.2014 Accepted 19.01.2015

Med Ultrason

2015, Vol. 17, No 1, 5-11

Corresponding author: Adriana Bințințan

$1^{\text {st }}$ Medical Clinic

3-5 Clinicilor street

400006, Cluj-Napoca, România

Phone: +40722329204

Fax: +40264590899

E-mail: abintintan@yahoo.com cidence of new varices is $7-8 \%$, with a similar rate of transition from small to large EV. The major risk that threatens the prognosis of a patient with EV is massive upper digestive bleeding, knowing that the first bleeding episode is associated with a $40 \%$ mortality rate [2]. The optimal interval for endoscopic surveilance in patients with liver cirrhosis depends on the ethiology and grade of EV; patients with EV grade I should be monitored endoscopically every 1-2 years, the short 1 year interval being recommended for patients with alcoholic cirrhosis; in patients without EV the endoscopic surveillance should be performed every 2-3 years to detect those with newly developed EV and hemorrhagic risk [3,4].

Esophagogastroduodenoscopy (EGD) remains the gold standard for diagnosis and grading of EV and for the evaluation of the risk of bleeding [5] but it has a series of 
dissadvantages that makes long term surveillance problematic: it is unpleasant for the patient and requires both complex logistics and qualified medical staff. Therefore, there is a strong need for another, less invasive set of investigations that have the ability to select patients with a higher risk of bleeding who will benefit from a therapeutic EGD, from those with low risk, who will not benefit at all.

There are already a number of non-invasive biochemical and imagistic investigations that are used to characterize the morphological changes and vascular flow pattern in the portal system in patients with liver cirrhosis and portal hypertension (PHT) such as transabdominal ultrasound (US) or the ultrasound study of liver stiffness (LSM) which are directly correlated with the severity of PHT [6-10].

Although there are publications in the literature showing that US is valuable in the screening of patients with $\mathrm{EV}$, the majority of studies are retrospective and have a low scientific yeld. There is a need for prospective well designed studies to investigate in depth this relationship.

The aim of our study is to analyze prospectively the role of Doppler US and LSM in the detection of newly developed EV or growth of already known EV in patients with liver cirrhosis.

\section{Material and metohd}

\section{Study design and patients}

This longitudinal prospective study was performed between 2009-2012 on patients evaluated at the $1^{\text {st }}$ Medical Clinic, Department of Gastroenterology, Emergency Clinical County Hospital Cluj-Napoca. The study protocol complies with the principles of the Helsinki Declaration and, together with the Informed Consent, was approved by the Ethical Commitee of the "Iuliu Hațieganu" University of Medicine and Pharmacy, Cluj-Napoca. All patients were informed regarding the objectives of our research and were asked to sign and date the Informed Consent Form.

Inclusion criteria: adult patients with viral, alcoholic or mixed type liver chirrosis confirmed either by liver biopsy or by clinical, biochemical and imaging criteria.

Exclusion criteria: adult patients with liver cirrhosis of another ethiology, patients under treatment with betablocking agents or antiviral therapy (interferon), patients with thrombosis of the portal vein system, history of upper digestive bleeding or history of transplantation, TIPS treatment or sclerosation of $\mathrm{EV}$, patients with concomitant hepatocarcinoma, presence of snon-PHT related splenomegaly, history of splenectomy and obesity with BMI $\geq 30 \mathrm{~kg} / \mathrm{m}^{2}$.

Patients included in the study were investigated as follows: clinical and biological evaluation, upper GI endoscopy, abdominal gray-scale and Doppler US and measurement of liver stiffness using uni-dimensional elastography after a fasting period of at least 8 hours and physical rest of $20 \mathrm{~min}$.

In patients with a higher risk of bias for LSM or Doppler US measurements due to perihepatic ascitis or ASAT, ALAT $\geq 100$ UI were evaluated only after the correction of these parameters, at a distance of 2-12 weeks from the inclusion in the study.

\section{Morphologic evaluation of the portal axis in gray-} scale abdominal ultrasound.

This evaluation was performed by one single experienced investigator using a General Electric Logiq S6 (General Electric Inc, NY, USA) device equipped with a convex probe $\mathrm{GE} 4 \mathrm{C}(2.5-5.5 \mathrm{MHz})$ and a linear probe GE 12L (7-12 MHz).

All measurements were performed using a standardized protocol: the long axis of the spleen was measured on the left mid-axillary line with the hilus of the spleen present on the screen and the diameter of the portal vein was measured in the hilus of the liver, $2 \mathrm{~cm}$ caudal from its bifurcation, as the patient was in deep inspiration. A value $\geq 13 \mathrm{~cm}$ for the spleen diameter was considered pathological.

\section{Assesment of blood flow pattern in the portal and splanhnic axis.}

Doppler US was performed using a low frequency of pulse repetition $(750-1200 \mathrm{~Hz})$; the wall filter was set between $0-50 \mathrm{~Hz}$; the color box was reduced to the minimum and colour amplification was adjusted to obtain a non-artefacted image.

The following Doppler parameters were recorded in all patients: maximal and mean velocity $\left(\mathrm{V}_{\text {maxpV }}, \mathrm{V}_{\mathrm{mPV}}\right)$, portal blood flow $\left(\mathrm{F}_{\mathrm{PV}}\right)$, hepatic artery resistivity index (HARI), hepatic artery pulsatility index (HAPI), hepatic blood flow $\left(\mathrm{F}_{\mathrm{HA}}\right)$, splenic artery resistivity index (SARI), splenic artery pulsatility index (SAPI) and splenic blood flow $\left(\mathrm{F}_{\mathrm{SA}}\right) . \mathrm{V}_{\text {max- }}$ $\mathrm{PV}, \mathrm{V}_{\mathrm{mPV}}$ and $\mathrm{F}_{\mathrm{PV}}$ were recorded at the level of the portal vein just before its bifurcation, with $\mathrm{a}<60^{\circ}$ angle correction, during inspiration. All measurements were performed at the level of the hepatic and splenic hilus, respectively.

Calculation of the specific indexes was done according to the following formulas:

Portal Hypertension Index: PHT Index $=($ HARI $x$ $0,69) \times($ SARI x 0,87$) / \mathrm{V}_{\mathrm{mPV}}[11]$

Doppler Perfusion Index: DPI $=\mathrm{F}_{\mathrm{HA}} /\left(\mathrm{F}_{\mathrm{HA}}+\mathrm{F}_{\mathrm{PV}}\right)$ [12] Hemodynamic liver index: $\mathrm{PVr} 1=\mathrm{PV}$ diameter $/ \mathrm{V}_{\mathrm{mPV}}$ [13]

Portal Vascular Resistance: PVR $=[(0,066 \times$ SAPI $)$ $0,044] \times \mathrm{F}_{\mathrm{PV}}[14]$

Spleno-Portal Index: SPI $=$ Splenic Index $/ \mathrm{V}_{\mathrm{m} \text { PV }}$ (Splenic Index $=$ long diameter of the spleen $\times$ short diameter of the spleen) [15]. 
Assesment of liver stiffness was randomly performed by two experienced investigators using the FibroScan $\AA$ (EchoSens, Paris, France) device equipped with a medium sized probe. For certification of a valid measurement we complied to the recomendations of the manufacturer and relied on the previous validated parameters: IQR (interquartile range) variation of 10 measurements compared to the mean value $<30 \%$ and the rate of success $>60 \%$ (number of valid measurements compared to the total number of measuments) $[16,17]$

Endoscopic evaluation of esophageal varices.

In all patients, an upper GI endoscopy was performed by a single investigator using the Olympus Exera II device. Four groups were created for statistical analysis: patients without EV (EV0) and with $\mathrm{EV}(\mathrm{EV}+)$, respectively small EV (EV0 and EV grade I) and large EV (EV grade II and III).

\section{Statistical analysis}

We used both descriptive and interferential statistics. For the descriptive variant, the indicators for centrality and dispersion were used: mean value and standard deviation for cases that have a gaussian distribution, respectively the $25 \%$ and $75 \%$ percentile for the variables that did not have a normal distribution of values. The interferential statistical evaluation aimed at assessing the concordances between the diagnostic test used and its golden standard equivalent.
The diagnostic accuracy was assessed by using the ROC curves (Receiver Operating Characteristic). Accordingly we calculated the optimal cut-off values for each parameter and various other statistical parameters: sensitivity (Se), specificity (Sp), accuracy, +LR, -LR, PPV, NPV, prevalence and the associated $95 \%$ confidence intervals.

For the studied parameters both the area under the ROC curve (AUROC) and the associated 95\% confidence interval were calculated.

The statistics were determined using the IBM SPSS v.21 version 3.03 software.

\section{Results}

General characteristics. The study group comprised 60 patients that fulfilled the inclusion criteria. The general and biochemical characteritics of these study groups are detailed in Table I.

Table II compares the grey-scale (US) and Doppler (USD) ultrasound in the 4 study groups: EV0, EV+, small EV and large EV.

Correlation between LSM and presence of $E V$. At a cut-off value of LSM $>15 \mathrm{kPa}$ has a remarkable AUROC of 0.96 with Se and Sp reaching $95.7 \%$ and respectively 100\% (table III).

Correlation between LSM and presence of large $\boldsymbol{E} \boldsymbol{V}$. For a cut-off value of $\mathrm{LSM}>28.80 \mathrm{kPa}$, the AUROC

Table I. Baseline clinical, biochemical and endoscopic characteristics of the study population (mean values \pm standard deviation)

\begin{tabular}{|c|c|c|c|c|c|}
\hline & Overall population $(n=60)$ & $\operatorname{EV0}(n=13)$ & $\mathrm{EV}+(\mathrm{n}=47)$ & small EV (n=28) & large $\mathrm{EV}(\mathrm{n}=32)$ \\
\hline Males, n (\%) & $39(65)$ & $7(17.9)$ & $32(82.1)$ & $16(41)$ & $23(59)$ \\
\hline Females, n (\%) & $21(35)$ & $6(28.6)$ & $15(71.4)$ & $12(57.1)$ & $9(42.9)$ \\
\hline Age (years) & $57.03 \pm 9.99$ & $52 \pm 5.33$ & $58.43 \pm 10.55$ & $52.75 \pm 7.36$ & $60.78 \pm 10.57$ \\
\hline BMI $(\mathrm{kg} / \mathrm{m} 2)$ & $26.99 \pm 4.34$ & $27.56 \pm 4.18$ & $26.84 \pm 4.41$ & $27.53 \pm 4.51$ & $26.53 \pm 4.20$ \\
\hline \multicolumn{6}{|l|}{ Etiology of cirrhosis, $\mathrm{n}(\%)$} \\
\hline Alcohol & $33(55)$ & $4(12.1)$ & $29(87.9)$ & $11(33.3)$ & $22(66.7)$ \\
\hline $\mathrm{C}$ virus & $17(28.3)$ & $6(35.3)$ & $11(64.7)$ & $10(58.8)$ & $7(41.2)$ \\
\hline $\mathrm{B}$ virus & $7(11.7)$ & $3(42.9)$ & $4(57.1)$ & $5(71.4)$ & $2(28.6)$ \\
\hline Mixt (B + alcohol) & $3(5)$ & $0(0)$ & $3(100)$ & $2(66.7)$ & $1(33,3)$ \\
\hline Child-Pugh A/B/C, n & $39 / 13 / 8$ & $13 / 0 / 0$ & $26 / 13 / 8$ & $28 / 0 / 0$ & $11 / 13 / 8$ \\
\hline MELD score & $11.22 \pm 4.08$ & $6.77 \pm 1.09$ & $12.45 \pm 3.72$ & $8.5 \pm 2.42$ & $13.59 \pm 3.74$ \\
\hline ALAT $(<55 \mathrm{U} / \mathrm{L})$ & $46.25 \pm 27.142$ & $66.69 \pm 29.73$ & $40.60 \pm 23.73$ & $56.43 \pm 30.99$ & $37.34 \pm 19.74$ \\
\hline ASAT (5-34 U/L) & $51.70 \pm 24.24$ & $49.92 \pm 24.54$ & $52.19 \pm 24.4$ & $52.71 \pm 25.63$ & $50.81 \pm 23.32$ \\
\hline $\operatorname{ALP}(98-279 \mathrm{U} / \mathrm{L}) *$ & 200 & 176 & 209 & 194.50 & 211 \\
\hline$\gamma \mathrm{GT}(7-32 \mathrm{U} / \mathrm{L})^{*}$ & 200 & 33 & 101 & 48.5 & 92 \\
\hline Bilirubin $(0-1.2 \mathrm{mg} / \mathrm{dL})^{*}$ & 1.3 & 0.98 & 1.5 & 1.14 & 1.55 \\
\hline Creatinine $(0.5-1.1 \mathrm{mg} / \mathrm{dL})$ & $0.88 \pm 0.22$ & $0.90 \pm 0.08$ & $0.87 \pm 0.25$ & $0.84 \pm 0.13$ & $0.90 \pm 0.28$ \\
\hline $\operatorname{INR}(0.8-1.2)$ & $1.29 \pm 0.30$ & $0.97 \pm 0.07$ & $1.38 \pm 0.28$ & $1.09 \pm 0.14$ & $1.46 \pm 0.30$ \\
\hline Platelets $\left(150-400 \times 10^{3} / \mathrm{mm}^{3}\right)$ & $136.32 \pm 75.60$ & $227 \pm 56.77$ & $111.23 \pm 59.36$ & $166.57 \pm 81.73$ & $109.84 \pm 59.24$ \\
\hline
\end{tabular}

$\mathrm{n}=$ number of patients, BMI = body mass index, MELD = model for end-stage liver disease,

ASAT $=$ aspartate aminotransferase, ALAT $=$ alanine aminotransferase, ALP $=$ alkaline phosphatases, $\gamma \mathrm{GT}=$ gammaglutamyl transpeptidase, $\mathrm{INR}=$ international normalised ratio,

* = median values - the variables did not have a Gaussian distribution 
Value of hepatic elastography and Doppler indexes for predictions of esophageal varices in liver cirrhosis

Table II. Baseline imagistic parameters of the study population (mean values \pm standard deviation)

\begin{tabular}{|c|c|c|c|c|c|}
\hline Imagistic parameters & Overall population $(n=60)$ & $\operatorname{EV0}(n=13)$ & $\mathrm{EV}+(\mathrm{n}=47)$ & $\operatorname{small} \operatorname{EV}(n=28)$ & large $\operatorname{EV}(n=32)$ \\
\hline $\mathrm{LSM}(\mathrm{kPa})$ & $35.35 \pm 20.73$ & $12.63 \pm 1.61$ & $41.63 \pm 19.09$ & $20.66 \pm 12.81$ & $48.20 \pm 17.99$ \\
\hline PV diameter (mm) & $12.68 \pm 1.79$ & $10.44 \pm 0.97$ & $13.30 \pm 1.43$ & $11.81 \pm 1.83$ & $13.45 \pm 1.38$ \\
\hline $\mathrm{V}_{\mathrm{mPV}}(\mathrm{cm} / \mathrm{sec})$ & $18.67 \pm 4.66$ & $20.16 \pm 4.61$ & $18.25 \pm 4.64$ & $19.61 \pm 4.45$ & $17.84 \pm 4.75$ \\
\hline $\mathrm{V}_{\operatorname{maxPV}}(\mathrm{cm} / \mathrm{sec})$ & $21.92 \pm 5.49$ & $22.56 \pm 5.12$ & $21.74 \pm 5.63$ & $22.58 \pm 4.56$ & $21.35 \pm 6.21$ \\
\hline $\mathrm{F}_{\mathrm{PV}}(\mathrm{ml} / \mathrm{min})$ & $710.22 \pm 258.61$ & $650 \pm 252.88$ & $879.46 \pm 215.24$ & $772.96 \pm 256.9$ & $655.32 \pm 251.2$ \\
\hline $\mathrm{F}_{\mathrm{HA}}(\mathrm{ml} / \mathrm{min})$ & $251.59 \pm 164.7$ & $207.5 \pm 139.33$ & $263.78 \pm 170.36$ & $220.10 \pm 123.2$ & $279.13 \pm 191.6$ \\
\hline $\mathrm{V}_{\mathrm{mHA}}$ & $23.02 \pm 10$ & $21.88 \pm 8.79$ & $23.34 \pm 10.53$ & $21.45 \pm 7.74$ & $25.04 \pm 12.46$ \\
\hline HARI & $0.85 \pm 0.16$ & $0.67 \pm 0.09$ & $0.73 \pm 0.09$ & $0.68 \pm 0.08$ & $0.75 \pm 0.09$ \\
\hline HAPI & $1.26 \pm 0.43$ & $1.13 \pm 0.28$ & $1.33 \pm 0.44$ & $1.19 \pm 0.28$ & $1.36 \pm 0.49$ \\
\hline A spl (cm2) & $76.42 \pm 36$ & $50.58 \pm 14.73$ & $83.59 \pm 36.93$ & $64.77 \pm 24.55$ & $86.55 \pm 41.36$ \\
\hline Pspl (cm) & $37.22 \pm 7.7$ & $30.91 \pm 4.67$ & $39.54 \pm 7.30$ & $36.10 \pm 7.96$ & $38.75 \pm 7.21$ \\
\hline Bipolar spleen diameter $(\mathrm{cm})$ & $146.10 \pm 33.27$ & $125.46 \pm 11.39$ & $151.81 \pm 35.10$ & $133.82 \pm 22.14$ & $156.84 \pm 37.74$ \\
\hline Splenic Index $(\mathrm{cm})^{*}$ & $60.47-108.16$ & $53.19-61.25$ & $70.23-117.70$ & $57.44-103.83$ & $70.23-134.75$ \\
\hline SARI & $0.93 \pm 0.81$ & $0.57 \pm 0.06$ & $0.84 \pm 0.91$ & $0.63 \pm 0.09$ & $0.92 \pm 1.11$ \\
\hline SAPI & $1.25 \pm 0.42$ & $0.84 \pm 0.91$ & $1.36 \pm 0.29$ & $1.07 \pm 0.32$ & $1.42 \pm 0.25$ \\
\hline Ascites (absent/ present), $\mathrm{n}$ & $35 / 25$ & $13 / 0$ & $22 / 25$ & $25 / 3$ & $10 / 22$ \\
\hline Collaterals (absent/present), $\mathrm{n}$ & $35 / 25$ & $13 / 0$ & $22 / 25$ & $20 / 8$ & $8 / 24$ \\
\hline
\end{tabular}

$\mathrm{n}=$ number of patients, LSM = Liver Stiffness Measurement, $\mathrm{PV}=$ portal vein, $\mathrm{V}_{\mathrm{mPV}}=$ portal blood mean velocity, $\mathrm{V}_{\operatorname{maxPV}}=$ portal blood maximum velocity, $\mathrm{F}_{\mathrm{PV}}=$ portal blood flow, $\mathrm{F}_{\mathrm{HA}}$ = hepatic blood flow, $\mathrm{V}_{\mathrm{mHA}}$ = hepatic artery blood mean velocity, HARI = hepatic artery resistance index, HAPI $=$ hepatic artery pulsatility index, $\mathrm{A} \mathrm{spl}=$ area of the spleen, $\mathrm{Pspl}=$ perimeter of the spleen, SARI=splenic artery resistance indices, SAPI $=$ splenic artery pulsatility indices, $*$ the $25-75$ percentile was noted

Table III. Performance of imagistic parameters for detection of presence of EV

\begin{tabular}{|c|c|c|c|c|c|c|c|}
\hline Paramater & & LSM (kPa) & DPI & PVr1 & PVR & SPI & PTH Index (m/s-1) \\
\hline Mean value & EV0 & $12.63 \pm 1.61$ & $0.24 \pm 0.15$ & $0.54 \pm 0.12$ & $9.42 \pm 7.5$ & $3.19 \pm 0.92$ & $1.23 \pm 0.511$ \\
\hline$\pm(\mathrm{SD})$ & $\mathrm{EV}+$ & $41.63 \pm 19.09$ & $0.26 \pm 0.11$ & $0.77 \pm 0.20$ & $32.42 \pm 16.9$ & $6.56 \pm 7.14$ & $2.17 \pm 2.2$ \\
\hline $\mathrm{p}$ & & $<0.001$ & 0.69 & $<0.001$ & $<0.0001$ & $<0.001$ & 0.001 \\
\hline Cut-offs & & $>15$ & $>0.103$ & $\geq 0.66$ & $>17.66$ & $>4.77$ & $>1.201$ \\
\hline $\mathrm{Se}(\%)$ & & 95 & 95 & 65 & 76 & 63 & 87 \\
\hline $\mathrm{Sp}(\%)$ & & 100 & 23 & 92 & 92 & 92 & 69 \\
\hline PPV (\%) & & 100 & 81 & 96 & 97 & 96 & 91 \\
\hline NPV (\%) & & 86 & 60 & 42 & 52 & 41 & 60 \\
\hline$+\mathrm{LR}$ & & - & 1.24 & 8.57 & 9.96 & 8.29 & 2.83 \\
\hline$-\mathrm{LR}$ & & 2.35 & 5.42 & 2.71 & 3.94 & 2.55 & 5.42 \\
\hline Ac $(\%)$ & & 96 & 80 & 71 & 80 & 70 & 83 \\
\hline AUC & & 0.96 & 0.54 & 0.83 & 0.90 & 0.85 & 0.79 \\
\hline $95 \% \mathrm{CI}$ & & 0.88-0.99 & $0.41-0.67$ & $0.72-0.92$ & $0.80-0.96$ & $0.74-0.93$ & $0.67-0.89$ \\
\hline K & & 0.90 & 0.24 & 0.41 & 0.40 & 0.38 & 0.41 \\
\hline
\end{tabular}

LSM = Liver Stiffness Measurement, DPI = Doppler Perfusion Index, PVr1= Hemodynamic, Liver Index, PVR= Portal Vascular Resistance, $\mathrm{SPI}=$ Spleno-Portal Index, PHT Index = Portal Hypertension Index, SD = standard deviations, EV $0 /+=$ without $/$ with esophageal varices, $\mathrm{Se}=$ sensitivity, $\mathrm{Sp}=$ specificity, $\mathrm{PPV}$ and $\mathrm{NPV}=$ positive and negative predictive values, $+\mathrm{LR}$ and $-\mathrm{LR}=$ positive and negative likehood ratio, $\mathrm{Ac}=$ accuracy, AUROC $=$ area under Receiver Operating Characteristic curve, $95 \% \mathrm{CI}=95 \%$ confidence interval, $\mathrm{K}=\mathrm{Cohen}$ 's coefficient of concordance Kappa

value reached a very good value of 0.9 , with Se and Sp of $87.2 \%$ and $82.76 \%$ respectively (table IV).

Correlation between USD and presence of $E V . P \mathrm{~V}_{\mathrm{r} 1}$ at a cut-off value $\geq 0.66$, has a good AUROC value of 0.83 , with $92 \% \mathrm{Sp}$ and $65 \%$ Se. For a cut-off value of PRV $>17.66$ the AUROC at 0.9 and Se at $92 \%$ are excellent. The SPI index at a cut-off value $>4.77$ has a good AUROC of 0.85 and excellent Sp of $92 \%$.

Correlation between USD and presence of large $\mathrm{EV}$. None of the Doppler indexes managed to offer significant results, the AUROC value being in all cases $\leq 0.75$, with a diagnostic accuracy ranging between $65-71 \%$ and low Se and $\mathrm{Sp}(<65.6 \%$ and $<78.6 \%$ respectively $)$.

\section{Disscusions}

Although the management of upper GI bleeding has improved in the last decade, the mortality rate associated with the first hemorrhagic episode still scores a high $20 \%$ value within the first 6 weeks [18]. This is a strong rea- 
Table IV. Performance of imagistic parameters for detection large EV

\begin{tabular}{|c|c|c|c|c|c|c|c|}
\hline Paramater & & LSM (kPa) & DPI & PVr1 & PVR & SPI & PTH Index (m/s-1) \\
\hline Mean value & EVs & $20.66 \pm 12.81$ & $0.23 \pm 0.12$ & $0.64 \pm 0.18$ & $24.37 \pm 21.4$ & $4.38 \pm 1.74$ & $0.01 \pm 0.005$ \\
\hline$\pm(\mathrm{SD})$ & EVL & $48.2 \pm 17.99$ & $0.27 \pm 0.12$ & $0.77 \pm 0.21$ & $29.48 \pm 15.3$ & $6.8 \pm 8.14$ & $0.02 \pm 0.02$ \\
\hline $\mathrm{p}$ & & $<0.001$ & 0.052 & 0.0001 & 0.0034 & $<0.0002$ & 0.0001 \\
\hline Cut-offs & & $>28.8$ & $>0.303$ & $>0.66$ & $>28.63$ & $>5.32$ & $>1.23$ \\
\hline $\mathrm{Se}(\%)$ & & 87.10 & 53.1 & 71.9 & 62.5 & 65,6 & 93.8 \\
\hline $\mathrm{Sp}(\%)$ & & 82.76 & 78.6 & 67.9 & 78.6 & 82.1 & 50 \\
\hline PPV (\%) & & 84.38 & 73 & 71 & 76 & 77 & 66 \\
\hline NPV (\%) & & 85.71 & 59 & 67 & 64 & 66 & 86 \\
\hline$+\mathrm{LR}$ & & 5.05 & 2.47 & 2.23 & 2.91 & 3.06 & 1.75 \\
\hline - LR & & 6.41 & 1.67 & 2.41 & 2.09 & 2.28 & 7.42 \\
\hline $\operatorname{Ac}(\%)$ & & 85 & 65 & 70 & 70 & 71 & 71 \\
\hline AUC & & 0.90 & 0.64 & 0.74 & 0.70 & 0.74 & 0.75 \\
\hline $95 \% \mathrm{CI}$ & & $0.77-0.95$ & $0.51-0.76$ & $0.61-0.85$ & $0.58-0.82$ & $0.61-0.85$ & $0.63-0.86$ \\
\hline K & & 0.7 & 0.31 & 0.39 & 0.40 & 0.43 & 0.41 \\
\hline
\end{tabular}

$\mathrm{EV}_{\mathrm{S}}=$ small esophageal varices, $\mathrm{EV}_{\mathrm{L}}=$ large esophageal varices, $\mathrm{LSM}=$ Liver Stiffness Measurement, DPI = Doppler Perfusion Index, $\mathrm{PVr}_{1}=$ Hemodynamic Liver Index, PVR= Portal Vascular Resistance, SPI = Spleno-Portal Index, PHT Index = Portal Hypertension Index, $\mathrm{SD}=$ standard deviations, $\mathrm{Se}=$ sensitivity, $\mathrm{Sp}=$ specificity, $\mathrm{PPV}$ and $\mathrm{NPV}=$ positive and negative predictive values, $+\mathrm{LR}$ and $-\mathrm{LR}=$ positive and negative likehood ratio, $\mathrm{Ac}=$ accuracy, $\mathrm{AUROC}=$ area under Receiver Operating Characteristic curve, $95 \% \mathrm{CI}=95 \%$ confidence interval, $\mathrm{K}=$ Cohen's coefficient of concordance Kappa

son to include patients with liver cirrhosis not known to have EV in programs of endoscopic screening. Patients with known EV also benefit from a timely diagnosis as, bleeding prophylaxis has been proven to reduce the risk of bleeding and death with as much as 50\% [19]. Although effective, the strategy of endoscopic screening in all cirrhotic patients is very expensive and suffers from the lack of compliance from patients. Moreover, there are recent studies which show that more than $50 \%$ of the screened cirrhotic patients will not develop EV within a period of 10 years follow-up [20]. In conclusion, there is a real need for complementary non-invasive diagnostic tools that can select for endoscopy only the subsets of patients that really need it.

A series of non-invasive tests have already been described in the literature as indicators of portal hemodynamics. A simple ennumeration shows how multiple and diverse these markers are: diameter of the portal vein, congestion index of the portal vein, velocity indexes and indexes for pulsatility and resistivity in the splanhnic vascular system, etc. However, none of them has managed to match EGD in terms of accuracy, sensibility and specificity. The battle is not lost though and we do believe that a combination of these parameters in complex diagnostic formulas could avoid the deficiencies of each particular parameter and lead to a more significant result that could eventually compete with EGD in terms of EV diagnosis.

We have chosen six imagistic parameters: LSM, DPI, $\mathrm{PV}_{\mathrm{r} 1}, \mathrm{PVR}, \mathrm{SPI}$ and PHT Index and analyzed them in the context of presence of EV and grade of EV (small or large).
In our study, Fibroscan had a high accuracy both for the prediction of $\mathrm{EV}+$ as well as the presence of large $\mathrm{EV}$. A value of LSM $>15 \mathrm{kPa}$ had a high accuracy for diagnosis of EV+ while a value exceeding $28.8 \mathrm{kPa}$ identified correctly patients with large EV.

These results are similar with the literature, the LSM cut-off value for diagnosis of EV+ ranging from 13.9 to $21.5 \mathrm{kPa}$. The sensitivity of elastography for the presence of EV has been high in the majority of the studies we have reviewed (76-95\%) but their corresponding specificity (43-78\%) was significantly lower than the value we obtained [9,21-26]. One explanation for this discordance may come from the fact that the reviewed studies were conducted on a population with a heterogenous ethiology of cirrhosis. Pritchatt et al [22] demonstrated that in the $\mathrm{HCV}$ group (viral C cirrhosis) the number of false negative cases was significantly higher than in the non-HCV ethiology group ( $0.6 \%$ vs. $10.3 \%)$ while Nguyen-Khac et al [27] proved that the cut-off value is higher in alcoholic liver cirrhosis $(19,5 \mathrm{kPa})$ compared with the $\mathrm{C}$ viral cirrhosis $(12,5-14,6 \mathrm{kPa})$.

Our study is not ethiology-homogenous but the predominant ethiology is alcoholic, in discordance with the published studies which have predominantly viral ethiology. Moreover, in our population, the viral ethiology is predominant in the non-EV group while alcoholics are more present in the EV group. The same distribution is also maintained in the small EV group (viral and alcoholic) vs the large EV group (mostly alcoholic). This distribution of ethiology may have had an impact on the final accuracy of our data. We have made an analysis of 
cut-off values on ethiologic subgroups but the reduced number of patients did not allow for conclusive results. However, when the entire group was analzyed, we obtained representative cut-off values for the presence of $\mathrm{EV}(15 \mathrm{kPa})$ and the diagnosis of large EV $(28.8 \mathrm{kPa})$.

Another source of bias that may explain our very high $\mathrm{Sp}$ values could have arisen because of the relatively low number of patients analyzed. The studies we have reviewed in the literature suffer from the same limitations, as the number of cases evaluated did not exceed 183 patients, the least number of cases (43 patients) being recorded in prospective studies [9]. Another limitation of our study and reason for our high Sp may arise from the numeric disparity between the EV0 $(n=13)$ and $\mathrm{EV}+(\mathrm{n}=37)$ groups. We could have increased the number of patients recruited in the EV0 group by relaxing the inclusion criteria but we did not want to lose the accuracy of our research protocol and therefore rejected this possibility.

In order to reach a numerical equilibrum between the study groups, we united the subgroups of patients without EV (EV0) and those with EV grade I in the small EV group which we compared with the large EV group created by the union of patients with EV grades II and III. In this setting we found a cut-off value of $28.8 \mathrm{kPa}$ which is within the limits of the literature (between $19.00 \mathrm{kPa}$ [21] and $30.5 \mathrm{kPa}$ [24]) and predicts the presence of large EV with good Se and Sp, and an excellent AUROC (0.9). Our results confirm the conclusions of Bonder et Afdhal [28] who recommended that all patients with liver cirrhosis and a value of LSM exceeding $15 \mathrm{kPa}$ should be screened for the detection of $\mathrm{EV}$ and those with a value over $20 \mathrm{kPa}$ should be treated with $\beta$-blocking agents.

The portal and splanhnic hemodynamics have been intensively studied to characterize the blood flow pattern in the portal system and its implications. Various indexes have been calculated using complex mathematical formulas in an attempt to characterize the blood flow pattern in the portal system: the congestion index of the portal vein, the spleno-portal index, the index of portal hypertension, the arterio-portal index, the indexes of pulsatility and resistivity in the hepatic and splenic arteries, etc. Of all these we chose to study in detail the correlation between the presence of EV and five indexes that contain in their formulas Doppler paramaters modified by the presence and the degree of PHT: DPI, PV $\mathrm{rl}$, PVR, SPI and PHT Index. We chose these indexes either because we considered them valuable for the prediction of EV (SPI, PHT Index) or because some of them have not been studied so far in relation to the presence of EV in cirrhotic patients or had not been validated on large cohorts of patients (DPI, $\mathrm{PV}_{\mathrm{r} 1}$ și PVR)
The value of Doppler indexes for detection of EV was modest in our study; only three of them achieved an AUROC higher than 0.8: $\mathrm{PV}_{\mathrm{rl}}, \mathrm{PVR}$ and SPI.

$\mathrm{PV}_{\mathrm{r} 1}$ has been most frequently studied as a non-invasive alternative to liver biopsy to predict progression towards cirrhosis, a value of $\mathrm{PVr}_{1}>0.07$ being proven to differentiate between chronic hepatitis and liver cirrhosis [13]. There is no report so far in the literature on the use of $\mathrm{PV}_{\mathrm{r} 1}$ for noninvasive prediction of $\mathrm{EV}$ presence and in this respect, our study has certain elements of originality. Our data shows that when $\mathrm{PV}_{\mathrm{r} 1} \geq 0.66$ the probability that $\mathrm{EV}$ are present is fairly high. However, $\mathrm{PV}_{\mathrm{r} 1}$ can not differentiate small $\mathrm{EV}$ from large EV and should not be used in this case.

Bolognesi et al has showned that PVR has a good accuracy in predicting the severity of PHT ( $\geq 10 \mathrm{mmHg}$ ) [14]. In our study PVR offered good prediction rates for the presence of EV but showed no value in differentiating small from large EV. As we are aware, this is the first study to evaluate the performance of PVR regarding the prediction of EV. Although, extrapolating from the study of Bolognesi, we would have expected to have significant results concerning the presence of large EV as well, the final data did not confirm this hypothesis.

The spleno-portal index (SPI) has been proposed for the first time by Liu et al in 2008 [15] to predict small and large EV. One of the advantages of SPI is that it can be recorded easily during the screening programme for the detection of liver hepatocarcinoma. At a cut-off value of 3 , Liu managed to predict the presence of $\mathrm{EV}$ in $92 \%$ of patients with Child A cirrhosis, avoiding thus an unpleasant diagnostic EGD [15]. Our results are also encouraging but the cut-off value for diagnosis of EV was higher, at 4.77. One explanation for this difference is that, unlike Liu, we included in our study patients not only with cirrhosis Child A ( $\mathrm{n}=39$ patients) but also with Child $\mathrm{B}$ or $\mathrm{C}(\mathrm{n}=21$ patients). When the results are extrapolated, we could speculate that a value of SPI above 3 has a high predictibility for the presence of EV in patients with Child A cirrhosis while a value of SPI over 4.77 is predictive irrespective of the grade of cirrhosis (Child A, B or C). In our evaluation SPI had no value in differentiating small EV from large EV.

The other parameters studied such as DPI and the PHT index have an AUROC value under 0.8 and therefore have been considered inadequate for the purpose of our study.

In conclusion we can say that Fibroscan has an important role in the prediction of presence of $\mathrm{EV}$, a value of LSM above $15 \mathrm{kPa}$ being suggestive in this respect while a value over $28 \mathrm{kPa}$ pointing towards the presence of large EV. Our study has also identified three Doppler indexes: $\mathrm{PVr}_{1}$, PVR și SPI which, at cut-off values of $0.66,17.66$ and 4.77 respectively can predict the pres- 
ence of EV in patients with liver cirrhosis. We consider that further studies, in a multicentric format and on larger cohorts of patients are required to confirm our results and to offer them a strong clinical significance. We are confident that the inclusion of the above discussed indexes in a diagnostic algorithm can reduce the need for endoscopic screening in patients with liver cirrhosis in the future.

\section{Conflict of interest: none}

\section{References}

1. Schepis $\mathrm{F}$, Cammà $\mathrm{C}$, Niceforo $\mathrm{D}$, et al. Witch pacients with cirrhosis should undergo endoscopic screening for esophageal varices detection? Hepatology 2001; 33: 333-338.

2. Giannini E, Botta F, Borro P, et al. Platelet count/spleen diameter ratio: proposal and validation of non-invasive parameter to predict the presence of oesophageal varices in patients with liver cirrhosis. Gut 2003; 52: 1200-1205.

3. de Franchis R, Baveno V Faculty. Revising consensus in portal hypertension: report of the Baveno $\mathrm{V}$ consensus workshop on methodology of diagnosis and therapy in portal hypertension. J Hepatol 2010; 53: 762-768.

4. Berzigotti A, Seijo S, Reverter E, Bosch J. Assessing portal hypertension in liver diseases. Expert Rev Gastroenterol Hepatol 2013; 7: 141-155.

5. de Franchis R. Evaluation and follow-up of patients with cirrhosis and oesophageal varices. J Hepatol 2003; 38: 361 363.

6. Vizzutti F, Arena U, Rega L, Pinzani M. Non invasive diagnosis of portal hypertension in cirrhotic patients. Gastroenterol Clin Biol 2008; 32: 80-87.

7. Lupşor M, Badea R, Stefănescu H, et al. Analysis of histopathological changes that influence liver stiffness in chronic hepatites C. Results from a cohort of 324. J Gastrointestin Liver Dis 2008; 17: 155-163.

8. Foucher J, Chanteloup E, Vergniol J, et al. Diagnosis of cirrhosis by transient elastography (FibroScan): a prospectiv study. Gut 2006; 55: 403-408.

9. Vizzutti F, Arena U, Romanelli RG, et al. Liver stiffness measurement predicts sever portal hypertension in patients with HCV-related cirrhosis. Hepatology 2007; 45: 12901297.

10. Bureau C, Metivier S, Peron JM. Prospectiv assessment of liver stiffness for the non-invasive prediction of portal hypertension. J Hepatol 2007; 46: S34.

11. Piscaglia F, Donati G, Serra C, et al. Value of splanchnic doppler ultrasound in the diagnosis of portal hypertension. Ultrasound Med Biol 2001; 27: 893-899.

12. Leen E, Goldberg JA, Anderson JR, et al. Hepatic perfusion in patients with liver metastases: comparison with those patients with cirrhosis. Gut 1993; 34: 554-557.

13. Iliopoulos P, Vlychou M, Margaritis V, et al. Gray and color Doppler ultrasonography in differentiation between chronic viral hepatitis and compensated early stage cirrhosis. J Gastrointestin Liver Dis 2007; 16: 279-286.
14. Bolognesi M, Sacerdoti D, Merkel C, Bombonato G, Gatta A. Noninvasive grading of the severity of portal hypertension in cirrhotic patients by echo-color-Doppler. Ultrasound Med Biol 2001; 27: 901-907.

15. Liu CH, Hsu SJ, Liang CC, et al. Esophageal varices: noninvasive diagnosis with duplex Doppler US in patients with compensated cirrhosis. Radiology 2008; 248: 132-139.

16. Castera L, Forns X, Alberti A. Non-invasive evaluation of liver fibrosis using transient elastography. J Hepatol 2008; 48: 835-847.

17. Kettaneh A, Marcellin P, Douvin C, et al. Features associated with success rate and performance of fibroscan measurements for the diagnosis of cirrhosis in $\mathrm{HCV}$ patients: a prospective study of 935 patients. J Hepatol 2007; 46: 628-634.

18. Carbonell N, Pauwels A, Serfaty L, Fourdan O, Levy VG, Poupon R. Improved surival after variceal bleeding in patients with cirrhosis over the past decades. Hepatology 2004; 40: 652-659.

19. Imperiale TF, Chalasani N. A meta-analysis of endoscopic variceal ligation for primary prophylaxis of esophageal variceal bleeding. Hepatology 2001; 33: 802-807.

20. Thabut D, Moreau R, Lebrec D. Screening for esophageal varices: endoscopy, other tools, or endoscopy and other tools. Hepatology 2008; 47: 1434-1436.

21. Kazemi F, Kettaneh A, N'Kontchou G, et al. Liver stiffness measurement selects patients with cirrhosis at risk of bearing large oesophageal varices. J Hepatol 2006; 45: 230-235

22. Pritchett S, Cardenas A, Manning D, Curry M, Afdhal NH. The optimal cut-off for predicting large oesophageal varices using transient elastography is disease specific. J Viral Hepat 2011; 18: e75-e80.

23. Bureau C, Metivier S, Peron JM, et al. Transient elastography accurately predicts presence of significant portal hypertension in patients with chronic liver disease. Aliment Pharmacol Ther 2008; 27: 1261-1268.

24. Castera L, Le Bail B, Roudot-Thoraval F, et al. Early detection in routine clinical practice of cirrhosis and oesophageal varices in chronic hepatitis $\mathrm{C}$ : comparison of transient elastography (FibroScan) with standard laboratory tests and non-invasive scores. J Hepatol 2009; 50: 59-68.

25. Malik R, Lai M, Sadiq A, et al. Comparison of transient elastography, serum markers and clinical signs for the diagnosis of compensated cirrhosis. J Gastroenterol Hepatol 2010; 25: 1562-1568.

26. Nguyen-Khac E, Saint-Leger P, Tramier B, Coevoet H, Capron D, Dupas JL. Noninvasive diagnosis of large esophageal varices by fibroscan: strong influence of the cirrhosis etiology. Alcohol Clin Exp Res 2010; 34: 1146-1153.

27. Nguyen-Khac E, Chatelain D, Tramier B, et al. Assessment of asymptomatic liver fibrosis in alcoholic patients using transient elastopraphy: a comparison with seven non-invasive biological assays. Aliment Pharmacol Ther 2008; 28: 1118-1198.

28. Bonder A, Afdhal N. Utilization of FibroScan in clinical practice. Curr Gastroenterol Rep 2014; 16: 372. 